\title{
BRANCHING RANDOM MOTIONS, NONLINEAR HYPERBOLIC SYSTEMS AND TRAVELLING WAVES
}

\author{
Nikita RatanOV ${ }^{1}$
}

\begin{abstract}
A branching random motion on a line, with abrupt changes of direction, is studied. The branching mechanism, being independent of random motion, and intensities of reverses are defined by a particle's current direction. A solution of a certain hyperbolic system of coupled non-linear equations (Kolmogorov type backward equation) has a so-called McKean representation via such processes. Commonly this system possesses travelling-wave solutions. The convergence of solutions with Heaviside terminal data to the travelling waves is discussed. The paper realizes the McKean's program for the Kolmogorov-Petrovskii-Piskunov equation in this case. The Feynman-Kac formula plays a key role.
\end{abstract}

Mathematics Subject Classification. 35L60, 60J25, 60J80, 60J85.

Received April 25, 2005. Revised August 20, 2005.

\section{INTRODUCTION}

Travelling waves for the semilinear heat equation

$$
\frac{\partial u}{\partial t}=\frac{1}{2} \frac{\partial^{2} u}{\partial x^{2}}+f(u)
$$

have been extensively studied beginning from the classic papers by Kolmogorov-Petrovskii-Piskunov [22] and Fisher [9] (see detailed review in [37]).

A travelling wave with velocity parameter $a$ is a solution of equation (1) of the form $u=w_{a}(x-a t)$. Here function $w_{a}$ has the limits $w_{a}(-\infty)=0, w_{a}(+\infty)=1$ and, clearly, solves the ordinary equation

$$
\frac{1}{2} w_{a}^{\prime \prime}+a w_{a}^{\prime}+f\left(w_{a}\right)=0
$$

Basically, under certain assumptions on the nonlinearity term $f(u)$ the existence and uniqueness of solution of the initial value problem for (1) are well-known. Moreover, this solution (at least with Heaviside data) converges to the travelling front. More precisely,

$$
u(x+m(t), t) \rightarrow w_{a_{*}}(x), \quad t \rightarrow \infty
$$

Keywords and phrases. Branching random motion, travelling wave, Feynman-Kac connection, non-linear hyperbolic system, McKean solution.

${ }^{1}$ Faculty of Economics, Rosario University, Cl. 14, No. 4-69, Bogotá, Colombia; nratanov@urosario.edu.co

(c) EDP Sciences, SMAI 2006 
with $a_{*}=\sqrt{2 f^{\prime}(1)}$ and with some centering term $m=m(t)$.

Since McKean [25,26] (see also [3-5]) the connection between equation (1) and branching diffusion processes is established and widely applied. This approach is motivated by the following representation. Let $L(t)$ be the position of the left-most particle of a branching Brownian motion and let $g(u)$ be a probability generating function of the branching rule. Then $u=u(x, t)=\mathbb{P}(L(t)<x)$ is a solution of equation (1) with Heaviside initial conditions

$$
\left.u\right|_{t=0}=\theta(x)= \begin{cases}1, & x>0 \\ 0, & x \leq 0\end{cases}
$$

and with $f(u)=\lambda(g(u)-u)$, where $\lambda$ is the branching intensity.

Equation (1) arises in physics (especially in combustion theory), chemical kinetics and in a various biological models for gene developments, population dynamics or nerve propagation (see, for instance, $[15,18,19,38,39]$ and references therein).

Nevertheless this approach has the evident shortages: diffusion particles have infinite velocities and so they lack inertia, directions of their motion in separated time intervals are independent. To remedy these "unphysical" features it is possible to introduce a similar model, which is based on a random motion with finite velocity.

This idea has recently been the object of renewed interest of physicists and mathematicians (see $[7,8,10,12-17,23,24,28,29,32])$. It is applied also to financial market models $[6,36]$.

To describe these treatments we begin with the so-called telegraph random motion (see $[11,20,21,38]$ ). We consider a particle, initially (at time $t=\tau$ ) situated at point $x \in(-\infty, \infty)$, which moves on a line $(-\infty, \infty)$ with constant velocity $c$. At time $\tau$ it chooses either initial direction with equal probability. Then it repeatedly takes an opposite direction at random instants $T_{1}, T_{2}, \ldots$, which form a Poisson flow. The state of the process at time $t$ is $(X(t), \sigma(t))$, where $X(t)$ is the current particle's position and $\sigma(t)= \pm c$ is its current velocity.

Further, we consider the particle, which commences the random motion $(X, \sigma)$ for an exponentially distributed holding time $S$ independent of $X$. At $S$, the particle splits into a random number of pieces (offsprings). These new particles continue along independent paths of this random motion starting at $X(S)$, and are subject to the same splitting rule as the original particle. After an elapsed time $t-\tau$ we have $n=n(t-\tau)$ particles located at $X_{1}(t), \ldots, X_{n}(t)$, where $n(t-\tau)$ is stochastic.

Write $\mathbb{P}_{+,(x, \tau)}$ and $\mathbb{P}_{-,(x, \tau)}$ (with associated expectations $\mathbb{E}_{ \pm,(x, \tau)}$ ) for the laws of this process when it starts at time $\tau$ forwards $(+)$ and, respectively, backwards $(-)$, from the initial position $X(\tau)=x$.

Denote

$$
\begin{aligned}
& u_{+}(x, \tau, t)=\mathbb{P}_{+,(x, \tau)}\left(X_{1}(t)>0, \ldots, X_{n}(t)>0\right), \\
& u_{-}(x, \tau, t)=\mathbb{P}_{-,(x, \tau)}\left(X_{1}(t)>0, \ldots, X_{n}(t)>0\right) .
\end{aligned}
$$

Using a standard renewal arguments we prove (see Th. 3.1) that the probabilities $u_{ \pm}=u_{ \pm}(x, \tau, t)$ solve the semilinear hyperbolic system

$$
\left\{\begin{array}{l}
-\frac{\partial u_{+}}{\partial \tau}-c \frac{\partial u_{+}}{\partial x}=\mu_{+}\left(u_{-}-u_{+}\right)-\lambda_{+} u_{+}+\lambda_{+} F_{+}\left(u_{+}, u_{-}\right), \\
-\frac{\partial u_{-}}{\partial \tau}+c \frac{\partial u_{-}}{\partial x}=\mu_{-}\left(u_{+}-u_{-}\right)-\lambda_{-} u_{-}+\lambda_{-} F_{-}\left(u_{+}, u_{-}\right)
\end{array} \quad \tau<t\right.
$$

with the terminal conditions

$$
\left.u_{+}\right|_{\tau \uparrow t}=\left.u_{-}\right|_{\tau \uparrow t}=\theta(x) .
$$

Here $\mu_{+}>0$ and $\mu_{-}>0$ are the intensities of reverses, $\lambda_{+}$and $\lambda_{-}$are the breeding rates of forward (+) and backward (-) moving particle respectively;

$$
F_{+}\left(u_{+}, u_{-}\right)=\sum_{j+l \geq 2, j, l \geq 0} \beta_{j l}^{+} u_{+}^{j} u_{-}^{l}, \quad F_{-}\left(u_{+}, u_{-}\right)=\sum_{j+l \geq 2, j, l \geq 0} \beta_{j l}^{-} u_{+}^{j} u_{-}^{l}
$$


are probability generating functions of breeding rule; $\beta_{j l}^{+}\left(\beta_{j l}^{-}\right)$denote the probability of $j$ forward and $l$ backward moving offsprings of a particle, which has forward (backward) direction at a splitting time. We assume that

$$
\sum_{j+l \geq 2, j, l \geq 0} j \beta_{j l}^{ \pm}<\infty, \quad \sum_{j+l \geq 2, j, l \geq 0} l \beta_{j l}^{ \pm}<\infty
$$

A weak solution of system (5)-(6) exists and it is unique (see Appendix B). Solution $u_{ \pm}, u_{ \pm}(x, \tau, t) \in[0,1]$ has discontinuities concentrated on characteristics $l_{ \pm}=\{x= \pm c(t-\tau)\}$ only. The probabilistic interpretation (3)-(4) motivates following properties of $u_{ \pm}$,

$$
\begin{aligned}
& u_{-} \equiv 0 \text { for } x \leq-c(t-\tau) \text { and } u_{-} \equiv 1 \text { for } x>c(t-\tau) \\
& u_{+} \equiv 0 \text { for } x \leq-c(t-\tau) \text { and } u_{+} \equiv 1 \text { for } x \geq c(t-\tau) .
\end{aligned}
$$

Moreover, jump values vanish:

$$
\begin{aligned}
u_{-}(-c(t-\tau)+0, \tau, t) & \equiv 0, \quad u_{+}(c(t-\tau)-0, \tau, t) \equiv 1, \\
u_{-}(c(t-\tau)-0, \tau, t) & \rightarrow 1, \quad u_{+}(-c(t-\tau)+0, \tau, t) \rightarrow 0,
\end{aligned}
$$

as $\tau \downarrow-\infty$. The latter is proved in Section 3 (see Cor. 3.3).

System (5) is repeatedly obtained from both a phenomenological viewpoint and irreversible thermodynamics arguments in the papers of Dunbar-Othmer [7], Dunbar [8], Horsthemke [16,17], Mendez-Camacho [28], Mendez-Compte [29], Othmer-Dunbar-Alt [32]. Many authors have studied the travelling wave-type solutions of (5) emphasizing for stability properties (see review by Fort and Mendez [10] and references therein). Nevertheless, convergence results of the form (2) are still unknown with exception of the very special case of $F_{+}=u_{+}^{2}, F_{-}=u_{-}^{2}$. This nonlinearity corresponds to the following breeding rule. Particles, once born, live forever. At each splitting time $S$ each particle gives birth only to one offspring at its own current position $X(S)$ and of its own current velocity $\sigma(S)$. The large time behaviour of solutions of the Cauchy problem for (5) (with $F_{+}=u_{+}^{2}, F_{-}=u_{-}^{2}$ ) researched in details in [23] from both probabilistic and analytic viewpoints (see also [24]).

In this paper we discuss much more general branching rules. The main objective is to study the asymptotic behaviour of probabilistically represented solutions (3)-(4) of (5)-(6) keeping our treatment in the framework of the following three-step McKean's program [25]:

1) proof of existence of limits

$$
u_{+}\left(x+m_{+}, \tau, t\right) \rightarrow w_{+}(x), \quad u_{-}\left(x+m_{-}, \tau, t\right) \rightarrow w_{-}(x), \quad \tau \downarrow-\infty
$$

for all $x, t \in(-\infty, \infty)$, where $m_{ \pm}$are the medians of $u_{ \pm}$;

2) stability properties of travelling fronts with respect to the velocity value;

3 ) identification of the limits in (11) as a travelling-wave solution of (5).

Notice that system (5) has two stationary solutions: $u_{+}=u_{-} \equiv 0$ and $u_{+}=u_{-} \equiv 1$. We assume that

(C1): there are no other stationary solutions of (5), i.e. the algebraic system

$$
\left\{\begin{array}{l}
\mu_{+}(y-x)+\lambda_{+}\left(F_{+}(x, y)-x\right)=0 \\
\mu_{-}(x-y)+\lambda_{-}\left(F_{-}(x, y)-y\right)=0
\end{array}\right.
$$

has no solutions $x, y$, such that $0 \leq x, y \leq 1$, excepting $\{0,0\}$ and $\{1,1\}$.

The result of the first step is the following theorem. 
Theorem 1.1. Let parameters $\lambda_{ \pm}, \mu_{ \pm}$and branching rules $F_{ \pm}$satisfy conditions $C 1$. Then the limits in (11) exist with the centering terms $m_{ \pm}=m_{ \pm}(\tau)$ which are defined so as to satisfy

$$
u_{+}\left(m_{+}, \tau, t\right)=u_{-}\left(m_{-}, \tau, t\right)=1 / 2
$$

As it follows from assertions (7)-(10), the centering terms $m_{ \pm}$in (12) are well defined. Theorem 1.1 is proved in Section 4.

We pass the second and the third steps assuming certain restrictions. To describe our assumptions we define the following expected numbers of particles born in each splitting:

$$
J_{11}=\sum j \beta_{j l}^{+}, \quad J_{12}=\sum l \beta_{j l}^{+}, \quad J_{21}=\sum j \beta_{j l}^{-}, \quad J_{22}=\sum l \beta_{j l}^{-} .
$$

Note that the matrix

$$
J=\left(\begin{array}{ll}
J_{11} & J_{12} \\
J_{21} & J_{22}
\end{array}\right)=\left(\begin{array}{ll}
\frac{\partial F_{+}}{\partial u_{+}} & \frac{\partial F_{+}}{\partial u_{-}} \\
\frac{\partial F_{-}}{\partial u_{+}} & \frac{\partial F_{-}}{\partial u_{-}}
\end{array}\right)_{\left.\right|_{u_{+}=u_{-}=1}}
$$

represents the Jacobian of nonlinearity $\left\{F_{+}\left(u_{+}, u_{-}\right), F_{-}\left(u_{+}, u_{-}\right)\right\}$at $\{1,1\}$.

Let

$$
\begin{gathered}
b_{11}=\mu_{+}+\lambda_{+}\left(1-J_{11}\right), \quad b_{22}=\mu_{-}+\lambda_{-}\left(1-J_{22}\right) \\
b_{12}=\mu_{+}+\lambda_{+} J_{12}, \quad b_{21}=\mu_{-}+\lambda_{-} J_{21} .
\end{gathered}
$$

We assume $b_{i j}, i, j=1,2$ satisfy the condition

(C2): $\quad b_{11}+b_{22}<2 \sqrt{b_{12} b_{21}}, \quad b_{22}>0$.

A travelling wave solution to (5) is a solution of the form $u_{+}=w_{+}(x-a(t-\tau)), u_{-}=w_{-}(x-a(t-\tau))$. Define

$$
a_{*}=c \frac{b_{11}^{2}-b_{22}^{2}+4 \sqrt{b_{12} b_{21}\left(b_{12} b_{21}-b_{11} b_{22}\right)}}{\left(b_{11}-b_{22}\right)^{2}+4 b_{12} b_{21}} .
$$

It can be proved that $0<a_{*}<c$ (see Prop. 5.1 below).

The following theorem is proved in Section 5.

Theorem 1.2. If conditions $C 1$ and $C 2$ hold and $a \in\left[a_{*}, c\right)$, then there exists one and, modulo translation, only one wave solution travelling with speed a.

In Section 6 we try to pass the third step of McKean's program. We prove that the limits in (11) form a properly shifted travelling-wave solution and we determine the value of this shift.

Theorem 1.3. Let conditions (C1) and (C2) hold and the limit

$$
\lim _{\tau \downarrow-\infty}\left(m_{-}(\tau)-m_{+}(\tau)\right)=\beta
$$

exists. Then

$$
\lim _{\tau \downarrow-\infty}\left(-\dot{m}_{+}(\tau)\right)=\lim _{\tau \downarrow-\infty}\left(-\dot{m}_{-}(\tau)\right)=a_{*} .
$$

Moreover, if $w_{+}=w_{+}(x), w_{-}=w_{-}(x)$ are the limits in (11), then $\left\{w_{+}=w_{+}(x), w_{-}^{*}=w_{-}(x-\beta)\right\}$ (or $\left.\left\{w_{+}^{*}=w_{+}(x+\beta), w_{-}=w_{-}(x)\right\}\right)$ form a (modulo translation unique) wave solution travelling with the velocity $a_{*}$.

Here $\dot{m}_{+}$and $\dot{m}_{-}$denote a derivatives in $\tau$. 
Remark 1.4. In this paper we explore the backward equations (5), but it is easy to transfer all results into the results for the forward equation

$$
\left\{\begin{array}{l}
\frac{\partial u_{+}}{\partial t}-c \frac{\partial u_{+}}{\partial x}=\mu_{+}\left(u_{-}-u_{+}\right)-\lambda_{+} u_{+}+\lambda_{+} F_{+}\left(u_{+}, u_{-}\right), \\
\frac{\partial u_{-}}{\partial t}+c \frac{\partial u_{-}}{\partial x}=\mu_{-}\left(u_{+}-u_{-}\right)-\lambda_{-} u_{-}+\lambda_{-} F_{-}\left(u_{+}, u_{-}\right),
\end{array} \quad t>\tau\right.
$$

The unique weak solution of system (14) (with Heaviside initial condition $\left.u_{ \pm}\right|_{t \downarrow \tau}=\theta(x)$ ) can be interpreted as

$$
u_{ \pm}(x, \tau, t)=\mathbb{P}_{ \pm,(0, \tau)}\left(X_{1}(t)<x, \ldots, X_{n}(t)<x\right)
$$

Remark 1.5. Recently some results on travelling waves for the branching telegraph-like processes with variable velocities (and for respective hyperbolic systems with variable coefficients $c=c(x)$ ) have been obtained (see [35]). These results are heavily based on theorems 1.1-1.3.

\section{Telegraph processes and Feynman-KaC COnNection}

In this section we remind some properties of the telegraph process (see [21] or [38] for further details).

Let $(\Omega, \mathcal{F}, \mathbb{P})$ be a complete probability space and let $(X(t), \sigma(t)), t \geq \tau$ be a telegraph process with alternating velocities $\pm c$ and intensities $\mu_{ \pm}$, which is defined in introduction. Denote by $f_{ \pm}$and $b_{ \pm}$(generalized) transition probability densities of Markov process $(X(t), \sigma(t)), t \geq \tau$, i.e. for any measurable $A$

$$
\begin{aligned}
& \mathbb{P}_{ \pm,(x, \tau)}(X(t) \in A, \sigma(t)=+c)=\int_{A} f_{ \pm}(x, \tau, y, t) \mathrm{d} y, \\
& \mathbb{P}_{ \pm,(x, \tau)}(X(t) \in A, \sigma(t)=-c)=\int_{A} b_{ \pm}(x, \tau, y, t) \mathrm{d} y,
\end{aligned}
$$

and denote $f=\left(f_{+}+f_{-}\right) / 2, \quad b=\left(b_{+}+b_{-}\right) / 2, \quad p_{+}=f_{+}+b_{+}, \quad p_{-}=f_{-}+b_{-}, \quad p=f+b=\left(p_{+}+p_{-}\right) / 2$. Observe that these functions contain Dirac component along characteristics (see e.g. [31]).

It is known that $\left(f_{+}, f_{-}\right),\left(b_{+}, b_{-}\right)$as well as $\left(p_{+}, p_{-}\right)$are solutions of the system

$$
\left\{\begin{array}{l}
-\frac{\partial v_{+}}{\partial \tau}-c \frac{\partial v_{+}}{\partial x}=\mu_{+}\left(v_{-}-v_{+}\right) \\
-\frac{\partial v_{-}}{\partial \tau}+c \frac{\partial v_{-}}{\partial x}=\mu_{-}\left(v_{+}-v_{-}\right), \quad \tau<t
\end{array}\right.
$$

To determine $f_{ \pm}, b_{ \pm}$or $p_{ \pm}$system (15) should be supplied with the following terminal conditions:

$$
\begin{aligned}
\left.f_{+}\right|_{\tau \uparrow t}=\delta(x-y), & \left.f_{-}\right|_{\tau \uparrow t}=0 ; \\
\left.b_{+}\right|_{\tau \uparrow t}=0, & \left.b_{-}\right|_{\tau \uparrow t}=\delta(x-y)
\end{aligned}
$$

and

$$
\left.p_{+}\right|_{\tau \uparrow t}=\delta(x-y),\left.\quad p_{-}\right|_{\tau \uparrow t} \delta(x-y) .
$$

Moreover, for any bounded left-continuous in $y$ and continuous in $t$ function $g=g(y, \sigma, t), \sigma= \pm c, t \geq \tau$, $y \in(-\infty, \infty)$ the expectations

$$
v_{ \pm}(x, \tau, t)=\mathbb{E}_{ \pm,(x, \tau)} g(X(t), \sigma(t), t)
$$

form the solution of system (15) with the terminal conditions

$$
\left.v_{+}\right|_{\tau \uparrow t}=g_{+}(x, t),\left.\quad v_{-}\right|_{\tau \uparrow t}=g_{-}(x, t) .
$$


Here and everywhere below we repeatedly use $g_{+}$for $g(\cdot,+c, \cdot)$ and $g_{-}$for $g(\cdot,-c, \cdot)$.

In the particular case of $\mu_{+}=\mu_{-}=\mu \equiv$ const, system (15) is equivalent to so-called telegraph equation:

$$
\frac{\partial^{2} v}{\partial \tau^{2}}-2 \mu \frac{\partial v}{\partial \tau}=c^{2} \frac{\partial^{2} v}{\partial x^{2}}
$$

where $v=\left(v_{+}+v_{-}\right) / 2$. This transition is known as the Kac trick [21].

Solutions of equations (15) and (16) are well defined with $\delta$-functions in terminal values (see e.g. [11], [31] or [33]). For example, the exact expressions for $f_{ \pm}, b_{ \pm}$are well known (see (A.2)-(A.3) and cf. [2]):

$$
\begin{gathered}
f_{+}=\mathrm{e}^{-\mu_{+}(t-\tau)} \delta(x+c(t-\tau))+E(x, \tau, t) I_{1}\left(\mu_{*} \sqrt{(t-\tau)^{2}-x^{2} / c^{2}}\right) \frac{\mu_{*}(t-\tau-x / c)}{\sqrt{(t-\tau)^{2}-x^{2} / c^{2}}}, \\
f_{-}=\mu_{-} E(x, \tau, t) I_{0}\left(\mu_{*} \sqrt{(t-\tau)^{2}-x^{2} / c^{2}}\right), \quad b_{+}=\mu_{+} E(x, \tau, t) I_{0}\left(\mu_{*} \sqrt{(t-\tau)^{2}-x^{2} / c^{2}}\right), \\
b_{-}=\mathrm{e}^{-\mu_{-}(t-\tau)} \delta(x-c(t-\tau))+E(x, \tau, t) I_{1}\left(\mu_{*} \sqrt{(t-\tau)^{2}-x^{2} / c^{2}}\right) \frac{\mu_{*}(t-\tau+x / c)}{\sqrt{(t-\tau)^{2}-x^{2} / c^{2}}},
\end{gathered}
$$

where $\mu_{*}=\sqrt{\mu_{+} \mu_{-}}, E(x, \tau, t)=\frac{1}{2 c} \mathrm{e}^{-\left(\mu_{+}+\mu_{-}\right)(t-\tau) / 2+\left(\mu_{+}-\mu_{-}\right) x /(2 c)}$.

Under rescaling $c \rightarrow \infty, \mu \rightarrow \infty$ such that $c^{2} / \mu \rightarrow$ const., equation (16) becomes backward Kolmogorov equation for the standard diffusion. More precisely the random motion $X=X(t)$ converges weakly to the Brownian motion (see, for instance, [20] or [34]). This observation motivates us to exploit this random process instead of Brownian motion.

We prove the existence of the limits in (11) by means of Feynman-Kac Lemma. To present this lemma in hyperbolic context let us consider the following linear terminal-value problem:

$$
\left\{\begin{array}{l}
-\frac{\partial v_{+}}{\partial \tau}-c \frac{\partial v_{+}}{\partial x}=\mu_{+}(x, \tau)\left(v_{-}-v_{+}\right)+k_{+}(x, \tau) v_{+}, \\
-\frac{\partial v_{-}}{\partial \tau}+c \frac{\partial v_{-}}{\partial x}=\mu_{-}(x, \tau)\left(v_{+}-v_{-}\right)+k_{-}(x, \tau) v_{-}, \quad \tau<t \\
\left.v_{+}\right|_{\tau \uparrow t}=g_{+}(x, t),\left.\quad v_{-}\right|_{\tau \uparrow t}=g_{-}(x, t) .
\end{array}\right.
$$

Here $k_{ \pm}=k_{ \pm}(x, \tau), \mu_{ \pm}=\mu_{ \pm}(x, \tau), \tau \leq t, x \in(-\infty, \infty)$ are functions with possible discontinuities concentrated on characteristics $x= \pm c(t-\tau) ; g_{ \pm}=g_{ \pm}(x, t)$ are bounded left-continuous in $x$ and continuous in $t, t \geq \tau$ functions. As before we repeatedly unite by $h(\cdot, \sigma, \cdot), \sigma= \pm c$ both $h_{+}$and $h_{-}$for all functions $h$ of this type.

A weak solution of (17) exists and it is unique (see e.g. [27]).

Let $X=X(t), t \geq \tau$ be the telegraph process with parameters $\mu_{ \pm}$, i.e. the transition probability densities $f_{ \pm}$and $b_{ \pm}$of $X$ satisfy (15). Let $\left\{v_{+}, v_{-}\right\}, v_{ \pm}=v_{ \pm}(x, \tau), \tau<t, x \in(-\infty, \infty)$ be a weak solution to (17).

Theorem 2.1 (Feynman-Kac connection). Let $\mathfrak{t}, \tau<\mathfrak{t}<t$ be a stopping time for $X$. Then $v_{+}$, $v_{-}$have the representation

$$
\begin{aligned}
& v_{+}(x, \tau)=\mathbb{E}_{+,(x, \tau)} v(X(\mathfrak{t}), \sigma(\mathfrak{t}), \mathfrak{t}) \exp \left(\int_{\tau}^{\mathfrak{t}} k(X(s), \sigma(s), s) \mathrm{d} s\right), \\
& v_{-}(x, \tau)=\mathbb{E}_{-,(x, \tau)} v(X(\mathfrak{t}), \sigma(\mathfrak{t}), \mathfrak{t}) \exp \left(\int_{\tau}^{\mathfrak{t}} k(X(s), \sigma(s), s) \mathrm{d} s\right) .
\end{aligned}
$$


Observe that for $\mathfrak{t}=t=$ const. formulas (18)-(19) connect $v_{ \pm}(\cdot, \tau)$ and $v_{ \pm}(\cdot, t)$ by means of the telegraph process $X=X(t)$. The original Feynman-Kac formula for the parabolic system exploits the Brownian motion for the analogous connection.

Proof. At first, let stopping time $\mathfrak{t}$ be a constant, $\mathfrak{t}=t, t>\tau$, and fixed.

Lemma 2.2. Functions $v_{ \pm}=v_{ \pm}(x, \tau)$, which are defined by (18)-(19), satisfy the following system of integral equations:

$$
\begin{aligned}
v_{+}(x, \tau)=v_{+}^{0}(x, \tau)+ & \int_{\tau}^{t} \mathrm{~d} s \int_{-\infty}^{\infty}\left[f_{+}(x, \tau, z, s) k_{+}(z, s) v_{+}(z, s)\right. \\
& \left.+b_{+}(x, \tau, z, s) k_{-}(z, s) v_{-}(z, s)\right] \mathrm{d} z \\
v_{-}(x, \tau)=v_{-}^{0}(x, \tau)+ & \int_{\tau}^{t} \mathrm{~d} s \int_{-\infty}^{\infty}\left[f_{-}(x, \tau, z, s) k_{+}(z, s) v_{+}(z, s)\right. \\
& \left.+b_{-}(x, \tau, z, s) k_{-}(z, s) v_{-}(z, s)\right] \mathrm{d} z
\end{aligned}
$$

where

$$
v_{ \pm}^{0}(x, \tau)=\mathbb{E}_{ \pm,(x, \tau)} g(X(t), \sigma(t), t) .
$$

The proof of Lemma follows form the evident identity: for any integrable function $\Phi$

$$
\exp \left(\int_{\tau}^{t} \Phi(s) \mathrm{d} s\right)=1+\int_{\tau}^{t} \Phi(s) \exp \left(\int_{s}^{t} \Phi(r) \mathrm{d} r\right) \mathrm{d} s .
$$

To finish the proof of Theorem 3.1 for a constant stopping time it is sufficient to apply $-\frac{\partial}{\partial \tau}-c \frac{\partial}{\partial x}$ to $(20)$ and $-\frac{\partial}{\partial \tau}+c \frac{\partial}{\partial x}$ to (21), exploiting (15) for $v_{ \pm}^{0}, f_{ \pm}$and $b_{ \pm}$. The passage to the general stopping time $\mathfrak{t}$ is plain (see e.g. $[30])$.

\section{Branching telegraph processes and MCKean Representation of solutions OF NONLINEAR HYPERBOLIC SYSTEMS}

Let the process $X=X(t)$ to be branching. Assume that the single particle starts at time $t=\tau$ from the point $x$ and performs the telegraph random motion. At exponentially distributed instant $S>\tau$ (with parameter $\lambda_{+}$ for a forward moving particle and with $\lambda_{-}$for a backward moving one) it splits into a several (random number) particles. The descendants start to move from the point $X(S)$ independently one from another. They in turn split and reverse by the same rule.

Suppose that the forward moving particle splits on $j$ forward and $l$ backward moving parts with probability $\beta_{j, l}^{+}, j+l \geq 2$. For the backward moving particle the respective probabilities are $\beta_{j, l}^{-}, j+l \geq 2$. Denote by $F_{+}\left(u_{+}, u_{-}\right)=\sum_{j+l \geq 2} \beta_{j, l}^{+} u_{+}^{j} u_{-}^{l}$ and $F_{-}\left(u_{+}, u_{-}\right)=\sum_{j+l \geq 2} \beta_{j, l}^{-} u_{+}^{j} u_{-}^{l}$ the probability generating functions of splitting rule.

As the result after an elapsed time $t-\tau>0$ we have $n$ particles situated at $X_{1}(t), \ldots, X_{n}(t)$ with the velocities $\sigma_{1}(t), \ldots, \sigma_{n}(t), n=n(t-\tau)$.

Consider the expectations

$$
\begin{aligned}
& u_{+}(x, \tau, t)=\mathbb{E}_{+,(x, \tau)} \prod_{i=1}^{n} g\left(X_{i}(t), \sigma_{i}(t), t\right), \\
& u_{-}(x, \tau, t)=\mathbb{E}_{-,(x, \tau)} \prod_{i=1}^{n} g\left(X_{i}(t), \sigma_{i}(t), t\right) .
\end{aligned}
$$


As before $g=g(x, \sigma, t), x \in(-\infty, \infty), \sigma= \pm c, t>\tau$ is a bounded left-continuous in $x$ and continuous in $t$ function.

The following theorem is well-known (see [12] and $c f$. [25]).

Theorem 3.1. Let $\left\{u_{+}, u_{-}\right\}, \tau<t, x \in(-\infty, \infty)$ be the unique bounded weak solution of system (5) with terminal conditions

$$
\left.u_{+}\right|_{\tau \uparrow t}=g_{+}(x, t),\left.\quad u_{-}\right|_{\tau \uparrow t}=g_{-}(x, t)
$$

(see Appendix B). Then $u_{+}, u_{-}$have representation (22)-(23).

Corollary 3.2. If $L(t)=\min _{1 \leq i \leq n} X_{i}(t)$ is the position of the left-most particle and $u_{ \pm}=u_{ \pm}(x, \tau, t)=$ $\mathbb{P}_{ \pm,(x, \tau)}(L(t)>0)$, then $u_{ \pm}$form the solution of (5) with the Heaviside terminal conditions

$$
\left.u_{ \pm}\right|_{\tau \uparrow t}=\theta(x)= \begin{cases}1, & x>0 \\ 0, & x \leq 0\end{cases}
$$

Corollary 3.3. The solution of problem (5), (25) satisfies the jump conditions (7)-(10).

Proof. Let us prove the assertion (10) for $u_{+}(-c(t-\tau)+0, \tau, t):=\psi_{+}(\tau)$. From Corollary 3.2 it follows that

$$
\psi_{+}(\tau-\Delta \tau)=\left(1-\mu_{+} \Delta \tau\right)\left(1-\lambda_{+} \Delta \tau\right) \psi_{+}(\tau)+\lambda_{+} \Delta \tau F_{+}\left(\psi_{+}(\tau), 0\right)+o(\Delta \tau), \Delta \tau \rightarrow 0
$$

which implies the differential equation

$$
\frac{\mathrm{d} \psi_{+}}{\mathrm{d} \tau}=\left(\mu_{+}+\lambda_{+}\right) \psi_{+}-\lambda_{+} F_{+}\left(\psi_{+}, 0\right), \tau<t,\left.\quad \psi_{+}\right|_{\tau \uparrow t}=1
$$

Hence $\psi_{+}(\tau) \leq \mathrm{e}^{-\left(\mu_{+}+\lambda_{+}\right)(t-\tau)} \rightarrow 0$, as $\tau \downarrow-\infty$. The complete proof is similar.

Following Horsthemke $[16,17]$ we consider three main types of splitting rules.

1. Isotropic reaction walk

Let $F_{+}=F_{-}=F(u)$, where $u=\left(u_{+}+u_{-}\right) / 2$ and $F(u)=\sum_{k \geq 2} \beta_{k} u^{k}$. This means that at breeding times a particle splits onto $k$ parts with the probability $\beta_{k}$, which does not depend on the direction of motion. New particles choose either direction with equal probability.

In the particular case of $\mu_{+}=\mu_{-}=\mu(\tau)$ and $\lambda_{+}=\lambda_{-}=\lambda$ ( $\lambda$ is a constant) one can obtain from (5)

$$
\left\{\begin{array}{l}
\frac{\partial u}{\partial \tau}+c \frac{\partial w}{\partial x}=-\lambda(F(u)-u) \\
\frac{\partial w}{\partial \tau}+c \frac{\partial u}{\partial x}=(2 \mu+\lambda) w, \quad \tau<t
\end{array}\right.
$$

$w=\left(u_{+}-u_{-}\right) / 2$. Eliminating $w$ we have

$$
\frac{\partial^{2} u}{\partial \tau^{2}}-2(\mu+\lambda) \frac{\partial u}{\partial \tau}=c^{2} \frac{\partial^{2} u}{\partial x^{2}}-\lambda \frac{\partial F(u)}{\partial \tau}+\left(\lambda^{2}+2 \mu \lambda\right)(F(u)-u) .
$$

Notice that (26) and (27) are equivalent to the reaction Cattaneo system and to the reaction telegraph equation respectively (see $[10,13,16,17]$ ). If $F(u)=u^{2}$, the hyperbolic version of the classical Kolmogorov-PetrovskiiPiskunov equation arises (see $[22,25])$. 
2. Direction independent reaction walk

Assume a particle does not die and at the exponentially distributed instant gives a birth to $k$ new particles (with probability $\beta_{k}$ ). Daughter particles choose either direction with equal probability and move accordingly with the same rule. In this case the nonlinearity of (5) has the form $F_{+}=F(u) u_{+}, F_{-}=F(u) u_{-}$, where $F(u)=\sum_{k \geq 1} \beta_{k} u^{k}$. For the so-called branching-coalescence direction independent kinetic scheme [17] $F(u)=u$.

If $\mu_{+}=\mu_{-}=\mu, \lambda_{+}=\lambda_{-}=\lambda$, then system (5) is equivalent to

$$
\left\{\begin{array}{l}
\frac{\partial u}{\partial \tau}+c \frac{\partial w}{\partial x}=\lambda u(F(u)-1), \\
\frac{\partial w}{\partial \tau}+c \frac{\partial u}{\partial x}=(2 \mu+\lambda) w-\lambda F(u) w, \quad \tau<t .
\end{array}\right.
$$

No reaction telegraph equation can be obtained in this case.

3. Direction dependent reaction walk

Consider the previous regime, but with some significant modifications. We shall distinguish two main versions.

A. Suppose that each new particle starts strictly in the opposite direction to the direction of the maternal particle. The generating functions are $F_{+}=F\left(u_{-}\right) u_{+}, F_{-}=F\left(u_{+}\right) u_{-}$. In the particular case $F(u)=u$ (i.e. only one new particle arises) we have the branching-coalescence direction dependent kinetic scheme (see [17]).

B. Assume that each new particle starts in the same direction the maternal particle currently moves. The generating functions now are $F_{+}=F\left(u_{+}\right) u_{+}, F_{-}=F\left(u_{-}\right) u_{-}$. The particular case of $F_{+}=F_{-}=u$ is researched in details by Lyne [23] (see also [24]).

\section{Kolmogorov-Petrovskim-Piskunov lemma FOR HyPerboliC Systems. Proof of TheOREM 1.1}

The following proposition plays a key role in the further construction.

Proposition 4.1. Let $\left\{u_{+}, u_{-}\right\}$be the McKean solution of (5) with Heaviside terminal data (25) at fixed time horizon $t_{0}$ :

$$
\left.u_{+}\right|_{\tau \uparrow t_{0}}=\left.u_{-}\right|_{\tau \uparrow t_{0}}=\theta(x) .
$$

Then functions $u_{+}\left(x+m_{+}(\tau), \tau, t_{0}\right)$ and $u_{-}\left(x+m_{-}(\tau), \tau, t_{0}\right)$ increase in $\tau$, if $x>0$, and decrease in $\tau$, if $x<0$. Here $m_{ \pm}=m_{ \pm}(\tau)$ are defined by (12).

Proof. (cf. Sect. 4 of [25]). First, we need in the following lemma.

Lemma 4.2. Let $\left\{v_{+}, v_{-}\right\}$be the solution to the Feynman-Kac system (17) with fixed time horizon $t_{0}$. If $v_{+}\left(x_{0}, \tau_{0}\right)>0$ or $v_{-}\left(x_{0}, \tau_{0}\right)>0, \tau_{0}<t_{0}$, then there exists sample path $X_{*}\left(x_{0}, t\right), t \in\left[\tau_{0}, t_{0}\right]$ of the telegraph process, such that

$$
v\left(X_{*}\left(x_{0}, t\right), \sigma_{*}\left(x_{0}, t\right), t\right)>0
$$

(here $\sigma_{*}\left(x_{0}, t\right)$ is the velocity of $\left.X_{*}\left(x_{0}, t\right)\right)$.

Proof (of Lemma). Suppose contrariwise, that the existence of $X_{*}$ fails. Define the stopping time $\mathfrak{t}$ so as to be the first solution in $t, t>\tau_{0}$ of

$$
v\left(X\left(x_{0}, t\right), \sigma\left(x_{0}, t\right), t\right) \leq 0 .
$$

The expectations in the Feynman-Kac formula (18)-(19) (with $x=x_{0}, \tau=\tau_{0}$ ) are nonpositive, while $v_{+}\left(x_{0}, \tau_{0}\right)>0$ or $v_{-}\left(x_{0}, \tau_{0}\right)>0$. This contradiction completes the proof.

Now we prove Proposition 4.1 for $u_{+}$. The proof for $u_{-}$is similar.

Fix $\tau_{0}<t_{0}$ and $\alpha>0$. Denote $x_{0}=m_{+}\left(\tau_{0}\right), x_{1}=m_{+}\left(\tau_{0}-\alpha\right)$. Set (omitting $t_{0}$ from the notations)

$$
\begin{aligned}
& V_{+}(x, \tau)=u_{+}\left(x+x_{1}, \tau-\alpha\right)-u_{+}\left(x+x_{0}, \tau\right) \\
& V_{-}(x, \tau)=u_{-}\left(x+x_{1}, \tau-\alpha\right)-u_{-}\left(x+x_{0}, \tau\right)
\end{aligned}
$$


We must prove that

$$
\begin{array}{ll}
V_{+}\left(x, \tau_{0}\right) \leq 0 & \text { for any } \quad x>0 \\
V_{+}\left(x, \tau_{0}\right) \geq 0 & \text { for any } \quad x<0 .
\end{array}
$$

First notice that for any $\bar{u}_{ \pm}, u_{ \pm} \in[0,1]$

$$
F_{ \pm}\left(\bar{u}_{+}, \bar{u}_{-}\right)-F_{ \pm}\left(u_{+}, u_{-}\right)=k_{1}^{ \pm} \cdot\left(\bar{u}_{+}-u_{+}\right)+k_{2}^{ \pm} \cdot\left(\bar{u}_{-}-u_{-}\right)
$$

where $k_{i}^{ \pm}, i=1,2$ are some positive analytic functions of $\bar{u}_{ \pm}, u_{ \pm}$. By (5) and (33) functions $V_{+}, V_{-}$form the solution of (17) with

$$
M_{+}=\mu_{+}+\lambda_{+} k_{2}^{+}, \quad M_{-}=\mu_{-}+\lambda_{-} k_{1}^{-}
$$

instead of $\mu_{+}, \mu_{-}$, and

$$
K_{+}=\lambda_{+}\left(k_{1}^{+}+k_{2}^{+}-1\right), \quad K_{-}=\lambda_{-}\left(k_{1}^{-}+k_{2}^{-}-1\right)
$$

instead of $k_{+}, k_{-}$.

From terminal conditions (28) it follows

$$
\begin{aligned}
& V_{ \pm}\left(x, t_{0}-0\right) \leq 0, \quad \text { if } \quad x>-x_{0} \\
& V_{ \pm}\left(x, t_{0}-0\right) \geq 0, \quad \text { if } \quad x<-x_{0} .
\end{aligned}
$$

To prove (31) suppose, contrariwise, that $V_{+}\left(x_{*}, \tau_{0}\right)>0$ for some $x_{*}>0$.

Lemma 4.3. If $V_{+}\left(x, \tau_{0}\right)>0$, then there exists a sample path $X_{*}=X_{*}\left(x_{*}, t\right)$ of the telegraph process which starts at $\left(x_{*}, \tau_{0}\right)$ and passes to $\left(x, t_{0}\right)$ with some $x<-x_{0}$, such that

$$
V\left(X_{*}\left(x_{*}, t\right),-\sigma_{*}\left(x_{*}, t\right), t\right)>0
$$

for all $t, \tau_{0} \leq t \leq t_{0}$.

Proof. Consider the system

$$
\left\{\begin{array}{l}
-\frac{\partial \bar{V}_{-}}{\partial \tau}-c \frac{\partial \bar{V}_{-}}{\partial x}=M_{+} \cdot\left(\bar{V}_{+}-\bar{V}_{-}\right)+K_{+} \cdot \bar{V}_{-} \\
-\frac{\partial \bar{V}_{+}}{\partial \tau}+c \frac{\partial \bar{V}_{+}}{\partial x}=M_{-} \cdot\left(\bar{V}_{-}-\bar{V}_{+}\right)+K_{-} \cdot \bar{V}_{+}, \tau<t_{0}
\end{array}\right.
$$

with the terminal conditions $\left.\bar{V}_{+}\right|_{\tau \uparrow t_{0}}=\left.V_{-}\right|_{\tau \uparrow t_{0}},\left.\bar{V}_{-}\right|_{\tau \uparrow t_{0}}=\left.V_{+}\right|_{\tau \uparrow t_{0}}$. Thus $\bar{V}(x, \sigma, t) \equiv V(x,-\sigma, t), t \in$ $\left[\tau_{0}, t_{0}\right], x \in(-\infty, \infty)$. Since system (4.3) has the Feynman-Kac form, by Lemma 4.2 there exists the sample path $X_{*}$ of the telegraph process with the property

$$
\bar{V}\left(X_{*}, \sigma_{*}, t\right)=V\left(X_{*},-\sigma_{*}, t\right)>0 .
$$

To finish the proof of Proposition 4.1 we fix the path $X_{*}$ and consider the telegraph process $X=X(0, t), t \in$ $\left[\tau_{0}, t_{0}\right]$ with starting point at 0 .

Let $\mathfrak{t} \in\left[\tau_{0}, t_{0}\right]$ be the first moment of intersection of $X$ with $X_{*}=X_{*}\left(x_{*}, t\right), \tau_{0}<t<t_{0}$. It is clear that at the passage time $\mathfrak{t}$ the trajectory $X_{*}$ continues backwards while $X$ has the forward direction, i.e. $\sigma_{*}\left(x_{*} \mathfrak{t}\right)=-c$, $\sigma(0, \mathfrak{t})=+c$. Applying Theorem 3.1 we have

$$
V_{+}\left(0, \tau_{0}\right)=\mathbb{E}_{+,\left(0, \tau_{0}\right)} V(X(\mathfrak{t}), \sigma(\mathfrak{t}), \mathfrak{t}) \exp \left(\int_{\tau_{0}}^{\mathfrak{t}} K(X(s), \sigma(s), s) \mathrm{d} s\right) .
$$


By (35) the expectation in (37) is positive while by the definition (30) we have $V_{+}\left(0, \tau_{0}\right)=0$. This contradiction completes the proof.

By Proposition 4.1 the following limits exist:

$$
\begin{aligned}
& \lim _{\tau \downarrow-\infty} u_{+}\left(x+m_{+}(\tau), \tau\right)=w_{+}(x), \\
& \lim _{\tau \downarrow-\infty} u_{-}\left(x+m_{-}(\tau), \tau\right)=w_{-}(x),
\end{aligned}
$$

which completes the proof of Theorem 1.1 .

Note that functions $w_{ \pm}=w_{ \pm}(x)$ increase in $x, w_{ \pm}(0)=1 / 2$ and by Lemma 4.1

$$
\begin{aligned}
& \frac{\partial u_{ \pm}}{\partial \tau}\left(x+m_{ \pm}(\tau), \tau\right) \leq 0 \quad \text { for } \quad x<0 \\
& \frac{\partial u_{ \pm}}{\partial \tau}\left(x+m_{ \pm}(\tau), \tau\right) \leq 0 \quad \text { for } \quad x>0
\end{aligned}
$$

Now we should establish a connection of $w_{+}$and $w_{-}$with travelling-wave solutions of (5). Our plan follows the strategy of McKean [25]. Firstly, we obtain some inequalities for possible velocities of travelling fronts (Sect. 5). Secondly, the upper bound for medians $m_{ \pm}$is found (Sect. 6). Finally, the direct analytic treatment leads to the main result.

\section{Wave solutions. Proof of Theorem 1.2}

In this section we study stability properties of travelling-wave solutions. We suppose here $\mu_{+}, \mu_{-}$to be constant. The travelling-wave solution of system (5) is a solution of the form $u_{ \pm}(x, \tau, t)=w_{ \pm}(x-a(t-\tau))$. Functions $w_{+}$and $w_{-}$describe a travelling wave, if

$$
\left\{\begin{array}{l}
-(c+a) w_{+}^{\prime}=\mu_{+}\left(w_{-}-w_{+}\right)-\lambda_{+} w_{+}+\lambda_{+} F_{+}\left(w_{+}, w_{-}\right), \\
(c-a) w_{-}^{\prime}=\mu_{-}\left(w_{+}-w_{-}\right)-\lambda_{-} w_{-}+\lambda_{-} F_{-}\left(w_{+}, w_{-}\right) .
\end{array}\right.
$$

We are interested in probabilistic solutions of $(42)$, i.e. $0 \leq w_{ \pm} \leq 1, \lim _{z \rightarrow-\infty} w_{ \pm}(z)=0, \lim _{z \rightarrow+\infty} w_{ \pm}(z)=1$.

The states $\{0,0\}$ and $\{1,1\}$ are clearly equilibriums of system (42). According with assumption C1 there are no other equilibrium points. We show in this section that condition $\mathrm{C} 2$ guarantees the point $\{0,0\}$ to be unstable and the point $\{1,1\}$ to be stable.

More precisely, we should prove, that there exists a monotone wave solution travelling with the speed $a$, $a_{*} \leq a<c$ from $\{0,0\}$ to $\{1,1\}$, where $a_{*}$ is some positive bound which depends on parameters $b_{i j}, i, j=1,2$ (see (13)). This solution $\left\{w_{+}, w_{-}\right\}$of $(42)$ is modulo translation unique.

The proof splits onto the following parts.

\subsection{Phase portrait at $\{0,0\}$}

A linearization of (42) at point $\{0,0\}$ has the form

$$
\left\{\begin{array}{l}
w_{+}^{\prime}=\frac{\lambda_{+}+\mu_{+}}{c+a} w_{+}-\frac{\mu_{+}}{c+a} w_{-} \\
w_{-}^{\prime}=\frac{\mu_{-}}{c-a} w_{+}-\frac{\lambda_{-}+\mu_{-}}{c-a} w_{-} .
\end{array}\right.
$$


Eigenvalues of (43) are the roots of the equation

$$
\zeta^{2}+\left[\frac{\lambda_{-}+\mu_{-}}{c-a}-\frac{\lambda_{+}+\mu_{+}}{c+a}\right] \zeta-\frac{\lambda_{+} \lambda_{-}+\mu_{+} \lambda_{-}+\mu_{-} \lambda_{+}}{c^{2}-a^{2}}=0 .
$$

Clearly, if $a^{2}<c^{2}$, the eigenvalues have opposite signs. After some easy algebra one can find that for a positive $\zeta$ eigenvector $\mathbf{e}=\left(e_{1}, e_{2}\right)$ satisfies $e_{1}>e_{2}>0$. Thus $\mathbf{e}$ is directed into $\left\{\left(w_{+}, w_{-}\right): 0<w_{-}<w_{+}\right\}$. Hence $\{0,0\}$ is a saddle point with positive outgoing orbit.

\subsection{Phase portrait at $\{1,1\}$}

The linear part of $(42)$ at $\{1,1\}$ has the matrix

$$
A=\left(\begin{array}{cc}
\frac{b_{11}}{c+a} & -\frac{b_{12}}{c+a} \\
\frac{b_{21}}{c-a} & -\frac{b_{22}}{c-a}
\end{array}\right),
$$

where $b_{i j}, i, j=1,2$ are defined in (13). Our aim is to show that if $a_{*}<a<c$ with a suitable $a_{*}>0$, then assumption $\mathrm{C} 2$ imply the state $\{1,1\}$ to be a stable node. Moreover eigenvectors of $A$ have positive entries.

To check this note that matrix $A$ has two negative eigenvalues if and only if

$$
\left\{\begin{array}{l}
\operatorname{tr} A<0, \\
\operatorname{det} A>0, \\
(\operatorname{tr} A)^{2}-4 \operatorname{det} A>0 .
\end{array}\right.
$$

Here $\operatorname{tr} A$ is the trace and $\operatorname{det} A$ is the $\operatorname{determinant}$ of matrix $A$.

Inequalities (44) read in details as follows:

$$
\begin{gathered}
\alpha B>b, \\
4 \mu_{*}^{2}>B^{2}-b^{2}, \\
f(\alpha) \equiv \alpha^{2}\left(b^{2}+4 \mu_{*}^{2}\right)-2 \alpha b B+B^{2}-4 \mu_{*}^{2}>0 .
\end{gathered}
$$

Here we use the following notations: $B=b_{11}+b_{22}, b=b_{11}-b_{22}, \mu_{*}^{2}=b_{12} b_{21}, \alpha=a / c$.

Proposition 5.1. Let condition C2 to be hold. Then (46) fulfilled and (47) is equivalent to $\alpha_{*}<\alpha<1$ with

$$
\alpha_{*}=\frac{b B+4 \mu_{*} \sqrt{\mu_{*}^{2}-b_{11} b_{22}}}{b^{2}+4 \mu_{*}^{2}} .
$$

Furthermore, $0<\alpha_{*}<1$ and inequality (45) holds for any $\alpha, \alpha_{*}<\alpha<1$.

Proof. First note that C2 leads to (46): if $B \geq 0$, then $4 \mu_{*}^{2}>B^{2}$ by the first part of C2 and thus we have (46); if $B<0$, then (by $b_{22}>0$ ) $b^{2}>B^{2}$ and (46) is obviously fulfilled.

Now we study the intersection of parabola (47) and the horizontal axis. Notice that $f(1)=4 b_{22}^{2}>0$ and $f(-1)=4 b_{11}^{2} \geq 0$ and, if $b B \neq 0$, then

$$
\begin{gathered}
f(b / B)=\left(B^{2}-b^{2}\right)\left(B^{2}-b^{2}-4 \mu_{*}^{2}\right) / B^{2}, \\
f(B / b)=4 \mu_{*}^{2}\left(B^{2} / b^{2}-1\right) .
\end{gathered}
$$

Furthermore, if $b_{11} \geq 0$, then $B^{2} \geq b^{2}$, and by (46) and (49) we have $f(b / B) \leq 0$; if $b_{11} \leq 0$, then $B^{2} \leq b^{2}$, and by $(50)$ it leads to $f(B / b) \leq 0$. Finally, in the case $b B=0$ by $(46)$ we have $f(0)<0$. 
Summarizing, we conclude that inequality (47) is equivalent to

$$
\alpha_{*}<\alpha<1,
$$

where $\alpha_{*}$ is the greater root of the equation $f(\alpha)=0$, i.e. $\alpha_{*}$ is defined by (48). Moreover, if $b_{11} \geq 0$, then $\alpha_{*}>b / B$, and if $b_{11} \leq 0$, then $\alpha_{*}>B / b$.

From C2 it follows that $\alpha_{*}>0$. Indeed, if $B>0$, then $f(0)=B^{2}-4 \mu_{*}^{2}<0$ and thus $\alpha_{*}$ is positive; if $B \leq 0$, then $b<0$ and thus by (50) $f(B / b)<0$, where $0<B / b<1$. Hence $\alpha_{*}>B / b>0$.

If condition $\mathrm{C} 2$ fails, then $\alpha_{*}$ can be negative. This case is not considered in this paper and will be elsewhere reported later.

To check (45) note that in the case $b_{11} \geq 0$ we have $B>0$, thus inequality (45) follows from $\alpha_{*}>b / B$. If $b_{11}<0$, then $b<0$ and for $B \leq 0$ we have $1>\alpha>\alpha_{*}>B / b$. Then $B>\alpha b$ and thus $\alpha B>\alpha^{2} b>b$, which is required. The case $b<0, B>0$ is evident.

Remark 5.2. It follows from our above explanations that as $\alpha_{*}$ is the greater root of $f(\alpha)=0$,

$$
B-\alpha_{*} b=2 \mu_{*} \sqrt{1-\alpha_{*}^{2}} .
$$

It is important to note that an eigenvectors of $A$ has the right entries.

Proposition 5.3. Let $\zeta$ be an eigenvalue of matrix $A$ and $\mathbf{e}=\left\{e_{1}, e_{2}\right\}$ be an eigenvector with the eigenvalue $\zeta$. If C2 holds, then $e_{1} e_{2}>0$.

Proof. The entries $e_{1}, e_{2}$ of e satisfy the equation

$$
\frac{b_{11} e_{1}-b_{12} e_{2}}{c+a}=\zeta e_{1}
$$

where

$$
\zeta=\frac{\operatorname{tr} A \pm \sqrt{(\operatorname{tr} A)^{2}-4 \operatorname{det} A}}{2}=\frac{1}{2\left(1-\alpha^{2}\right) c}[b-\alpha B \pm \sqrt{f(\alpha)}] .
$$

Therefore

$$
[B-\alpha b \pm \sqrt{f(\alpha)}] e_{1}=2(1-\alpha) b_{12} e_{2}
$$

We can note that

$$
B-\alpha b>0 .
$$

Indeed, if $b \geq 0$, then this inequality follows from (45). For $b<0$ and $B \leq 0$ we obtained (53) above (see the last paragraph of the proof of Proposition 4.1). In the case $b<0$ and $B>0$ inequality (53) is evident. Hence $B-\alpha b \pm \sqrt{f(\alpha)}=B-\alpha b \pm \sqrt{(B-\alpha b)^{2}-4 \mu_{*}^{2}\left(1-\alpha^{2}\right)}>0$ and the proposition follows from $b_{12}>0$.

If $\alpha=\alpha_{*}$, the negative double eigenvalue $\zeta=\frac{b-\alpha B}{2\left(1-\alpha^{2}\right) c}<0$ arises. Clearly, as $\alpha$ decreases through $\alpha_{*}$ the two negative eigenvalues $\zeta_{1}, \zeta_{2}<0$ of $A$ coalesce and, at least for $\alpha$ sufficiently close to $\alpha_{*}$, become a complex conjugate pair with negative real part. This corresponds to an eigenvector $\mathbf{e}=\left(e_{1}, e_{2}\right)$ with correct signs of entries:

$$
e_{2} / e_{1}=\frac{B-\alpha b}{2(1-\alpha) b_{12}}>0 .
$$

Notice that by the first equation of (42) one can see, that $\left.w_{+}^{\prime}\right|_{\left\{0 \leq w_{+} \leq 1, w_{-}=0\right\}}>0,\left.w_{+}^{\prime}\right|_{\left\{w_{+}=1,0 \leq w_{-} \leq 1\right\}}>0$, $\left.w_{+}^{\prime}\right|_{\left\{0 \leq w_{+}=w_{-} \leq 1\right\}}>0$. Hence the travelling wave solution can not leave the square $[0,1] \times[0,1]$.

This completes the proof of Theorem 1.2.

It is interesting to interpret these results for the main examples which were introduced in Section 3. 


\subsection{Examples}

We suppose here that $\mu_{+}=\mu_{-}=\mu>0$ and $\lambda_{+}=\lambda_{-}=\lambda>0$.

1. Isotropic reaction walk

Assume that $F_{+}=F_{-}=F(u), u=\frac{u_{+}+u_{-}}{2}$. Let $F^{\prime}(1)=\sum k \beta_{k}=q>1$ be the expected number of descendants in a single birth. We have $J_{11}=J_{12}=J_{21}=J_{22}=q / 2$ and $b_{11}=b_{22}=\mu+\lambda(1-q / 2)$, $b_{12}=b_{21}=\mu+\lambda q / 2$.

In this example condition $\mathrm{C} 2$ reads

$$
2 \mu>\lambda(q-2)
$$

(it disappears, if $q \leq 2$ ) and the critical velocity value is

$$
\alpha_{*}=\frac{\sqrt{\lambda(2 \mu+\lambda)(q-1)}}{\mu+\lambda q / 2} .
$$

2. Direction independent reaction walk

The reaction terms are $F_{+}=F(u) u_{+}$and $F_{-}=F(u) u_{-}$. In this case $J_{11}=J_{22}=1+q / 2, J_{12}=J_{21}=q / 2$. Here and below in the third example $q=F^{\prime}(1)>0$ is the mean number of descendants (maternal particle is not taking into account). So $b_{11}=b_{22}=\mu-\lambda q / 2, b_{12}=b_{21}=\mu+\lambda q / 2$ and thus C2 means $2 \mu>\lambda q$. The critical velocity value is

$$
\alpha_{*}=\frac{\sqrt{2 \lambda \mu q}}{\mu+\lambda q / 2} .
$$

3. Direction dependent reaction walk

For the version A we supposed $F_{+}=F\left(u_{-}\right) u_{+}$and $F_{-}=F\left(u_{+}\right) u_{-}$. Hence $J_{11}=J_{22}=1, J_{12}=J_{21}=q$. Thus $b_{11}=b_{22}=\mu, b_{12}=b_{21}=\mu+\lambda q$. In this case we have

$$
\alpha_{*}=\frac{\sqrt{\lambda q(2 \mu+\lambda q)}}{\mu+\lambda q} .
$$

The critical values of velocities of travelling waves (54)-(56) coincide with respective estimations for similar models due to Mendez et al. [28,29] and Horsthemke [17].

For the version B (where $F_{+}=F\left(u_{+}\right) u_{+}, F_{-}=F\left(u_{-}\right) u_{-}$, thus $J_{11}=J_{22} 1+q, J_{12}=J_{21}=0$ and $\left.b_{11}=b_{22}=\mu-\lambda q, b_{12}=b_{21}=\mu\right)$ in the same manner as before one can obtain

$$
\mu>\lambda q, \quad \alpha_{*}=\frac{\sqrt{\lambda q(2 \mu-\lambda q)}}{\mu}
$$

(cf. Lyne [23]).

Remark 5.4. Observe that under the standard scaling

$$
c, \mu \rightarrow \infty, \quad c^{2} / \mu \rightarrow 1
$$

system (5) is equivalent to nonlinear heat equation (1). The critical wave speed in the case of $(1)$ is $\alpha_{*}=\sqrt{2 f^{\prime}(1)}$ [22]. In the McKean's interpretation $f^{\prime}(1)=\lambda(Q-1)$, where $\lambda$ is the intensity of the birth process, $Q$ is the expected number of descendants in a single birth.

In the hyperbolic model all four formulas (54)-(57) lead to the same result:

$$
\alpha_{*} c \rightarrow \sqrt{2 \lambda(Q-1)} .
$$




\section{UPPER BOUND FOR MEDIANS AND CONVERGENCE TO TRAVELLING WAVES}

Fix time horizon $t$. To obtain an upper bound of $m_{ \pm}(\tau)$ we use comparison arguments and the results of Appendix A.

Theorem 6.1. Let condition C2 to be hold. Then functions $m_{ \pm}(\tau)$ satisfy the following inequalities

$$
m_{ \pm}(\tau) \leq \alpha_{*} c(t-\tau)-\gamma \ln (t-\tau)
$$

where $\alpha_{*}$ is defined by (48) and $\gamma$ is some positive constant.

Proof. Functions $u_{+}=\mathbb{P}_{+,(x, \tau)}(L(t)>0)$ and $u_{-}=\mathbb{P}_{-,(x, \tau)}(L(t)>0)$, where $L(t)$ is the position of the left-most particle, satisfy system (5) with Heaviside terminal conditions $\left.u_{ \pm}\right|_{\tau \uparrow t}=\theta(x)$. Hence $\bar{u}_{ \pm}=1-u_{ \pm}=$ $\mathbb{P}_{ \pm,(x, \tau)}(L(t) \leq 0)$ solve the system

$$
\left\{\begin{array}{l}
-\frac{\partial \bar{u}_{+}}{\partial \tau}-c \frac{\partial \bar{u}_{+}}{\partial x}=-b_{11} \bar{u}_{+}+b_{12} \bar{u}_{-}-\lambda_{+} R_{+}\left(\bar{u}_{+}, \bar{u}_{-}\right) \\
-\frac{\partial \bar{u}_{-}}{\partial \tau}+c \frac{\partial \bar{u}_{-}}{\partial x}=b_{21} \bar{u}_{+}-b_{22} \bar{u}_{-}-\lambda_{-} R_{-}\left(\bar{u}_{+}, \bar{u}_{-}\right) \\
\tau<t, \quad x \in(-\infty,+\infty)
\end{array}\right.
$$

with the terminal data

$$
\left.\bar{u}_{ \pm}\right|_{\tau \uparrow t}=\theta(-x), \quad x \in(-\infty, \infty) .
$$

Here $b_{i j}, i, j=1,2$ are defined in (13) and

$$
\begin{aligned}
& R_{+}(x, y) \equiv F_{+}(1-x, 1-y)+J_{11} x+J_{12} y-1, \\
& R_{-}(x, y) \equiv F_{-}(1-x, 1-y)+J_{21} x+J_{22} y-1 .
\end{aligned}
$$

Notice that, due to convexity of $F_{ \pm}$(see e.g. [1]), functions $R_{ \pm}(x, y) \geq 0$ for $(x, y) \in[0,1] \times[0,1]$.

Let $\bar{v}_{ \pm}$solve the respective linear system

$$
\left\{\begin{array}{l}
-\frac{\partial \bar{v}_{+}}{\partial \tau}-c \frac{\partial \bar{v}_{+}}{\partial x}=-b_{11} \bar{v}_{+}+b_{12} \bar{v}_{-}, \\
-\frac{\partial \bar{v}_{-}}{\partial \tau}+c \frac{\partial \bar{v}_{-}}{\partial x}=b_{21} \bar{v}_{+}-b_{22} \bar{v}_{-}, \quad \tau<t, \quad x \in(-\infty,+\infty)
\end{array}\right.
$$

with the same terminal conditions.

Thus $\bar{u}_{+} \leq \bar{v}_{+}$and $\bar{u}_{-} \leq \bar{v}_{-}$. Now to finish the proof it is sufficient to note that from Proposition A.2 it follows

as $\tau \downarrow-\infty$.

Notice that (58) implies

$$
\bar{v}_{ \pm}\left(\alpha_{*} c(t-\tau)-\gamma \ln (t-\tau), \tau, t\right) \rightarrow 0
$$

$$
\limsup _{\tau \downarrow-\infty}\left[-\dot{m}_{ \pm}\right] \leq \alpha_{*} c .
$$

Recall that here and everywhere below $\dot{m}=\mathrm{d} m / \mathrm{d} \tau$.

Fix $t \in(-\infty, \infty)$ and consider functions $U_{ \pm}$and $U_{ \pm}^{*}$ of the following form:

$$
\begin{aligned}
& U_{+}(x, \tau)=u_{+}\left(x+m_{+}, \tau, t\right), \quad U_{-}(x, \tau)=u_{-}\left(x+m_{-}, \tau, t\right), \\
& U_{+}^{*}(x, \tau)=u_{+}\left(x+m_{-}, \tau, t\right), \quad U_{-}^{*}(x, \tau)=u_{-}\left(x+m_{+}, \tau, t\right) .
\end{aligned}
$$

Clearly, $U_{+}(0, \tau)=U_{-}(0, \tau)=1 / 2$. 
In these notations system (5) leads to

$$
\left\{\begin{array}{l}
-\frac{\partial U_{+}}{\partial \tau}-\left(c-\dot{m}_{+}\right) \frac{\partial U_{+}}{\partial x}=\mu_{+}\left(U_{-}^{*}-U_{+}\right)+\lambda_{+}\left(F_{+}\left(U_{+}, U_{-}^{*}\right)-U_{+}\right) \\
-\frac{\partial U_{-}}{\partial \tau}+\left(c+\dot{m}_{-}\right) \frac{\partial U_{-}}{\partial x}=\mu_{-}\left(U_{+}^{*}-U_{-}\right)+\lambda_{-}\left(F_{-}\left(U_{+}^{*}, U_{-}\right)-U_{-}\right) \\
-\frac{\partial U_{+}^{*}}{\partial \tau}-\left(c-\dot{m}_{-}\right) \frac{\partial U_{+}^{*}}{\partial x}=\mu_{+}\left(U_{-}-U_{+}^{*}\right)+\lambda_{+}\left(F_{+}\left(U_{+}^{*}, U_{-}\right)-U_{+}^{*}\right) \\
-\frac{\partial U_{-}^{*}}{\partial \tau}+\left(c+\dot{m}_{+}\right) \frac{\partial U_{-}^{*}}{\partial x}=\mu_{-}\left(U_{+}-U_{-}^{*}\right)+\lambda_{-}\left(F_{-}\left(U_{+}, U_{-}^{*}\right)-U_{-}^{*}\right) .
\end{array}\right.
$$

The following theorem gives a simple sufficient condition for a convergence of $U_{ \pm}$and $U_{ \pm}^{*}$ to travelling waves. Denote $\psi(\tau)=m_{-}(\tau)-m_{+}(\tau)$.

Theorem 6.2. If the limit

exists, then there exist the limits

$$
\lim _{\tau \downarrow-\infty} \psi(\tau)=\beta
$$

$$
\lim _{\tau \downarrow-\infty}\left(-\dot{m}_{+}(\tau)\right)=a_{+}, \quad \lim _{\tau \downarrow-\infty}\left(-\dot{m}_{-}(\tau)\right)=a_{-},
$$

$a_{+}=a_{-}$and

$$
\lim _{\tau \downarrow-\infty} U_{+}^{*}(x, \tau)=w_{+}(x+\beta), \lim _{\tau \downarrow-\infty} U_{-}^{*}(x, \tau)=w_{-}(x-\beta) .
$$

Moreover, pair $\left\{w_{+}(x), w_{-}(x-\beta)\right\}$ (and $\left.\left\{w_{+}(x+\beta), w_{-}(x)\right\}\right)$ form a travelling-wave solution.

Proof. First note that by (38)-(39) and (62) the following limits exist

$$
\begin{aligned}
\lim _{\tau \downarrow-\infty} U_{+}(x, \tau)=w_{+}(x), & \lim _{\tau \downarrow-\infty} U_{-}(x, \tau)=w_{-}(x), \\
\lim _{\tau \downarrow-\infty} U_{+}^{*}(x, \tau)=w_{+}(x+\beta), & \lim _{\tau \downarrow-\infty} U_{-}^{*}(x, \tau)=w_{-}(x-\beta) .
\end{aligned}
$$

Integrating the first two equations of (61) in $\tau$ from $\tau-1$ to $\tau$ and in $x$ from 0 to $x$ and passing to the limit as $\tau \downarrow-\infty$ we obtain

$$
\left\{\begin{array}{l}
-\left(c+a_{+}\right)\left(w_{+}(x)-1 / 2\right)=\int_{0}^{x}\left[\mu_{+}\left(w_{-}\left(x^{\prime}-\beta\right)-w_{+}\left(x^{\prime}\right)\right)\right. \\
\left.+\lambda_{+}\left(F_{+}\left(w_{+}\left(x^{\prime}\right), w_{-}\left(x^{\prime}-\beta\right)\right)-w_{+}\left(x^{\prime}\right)\right)\right] \mathrm{d} x^{\prime}, \\
\left(c-a_{-}\right)\left(w_{-}(x)-1 / 2\right)=\int_{0}^{x}\left[\mu_{-}\left(w_{+}\left(x^{\prime}+\beta\right)-w_{-}\left(x^{\prime}\right)\right)\right. \\
\left.+\lambda_{-}\left(F_{-}\left(w_{+}\left(x^{\prime}+\beta\right), w_{-}\left(x^{\prime}\right)\right)-w_{-}\left(x^{\prime}\right)\right)\right] \mathrm{d} x^{\prime} .
\end{array}\right.
$$

Similarly, from the last two equations of $(61)$ it follows

$$
\left\{\begin{array}{l}
-\left(c+a_{-}\right)\left(w_{+}(x+\beta)-w_{+}\left(x_{0}+\beta\right)\right)=\int_{x_{0}}^{x}\left[\mu_{+}\left(w_{-}\left(x^{\prime}\right)-w_{+}\left(x^{\prime}+\beta\right)\right)\right. \\
\left.+\lambda_{+}\left(F_{+}\left(w_{+}\left(x^{\prime}+\beta\right), w_{-}\left(x^{\prime}\right)\right)-w_{+}\left(x^{\prime}+\beta\right)\right)\right] \mathrm{d} x^{\prime} \\
\left(c-a_{+}\right)\left(w_{-}(x-\beta)-w_{-}\left(x_{0}-\beta\right)\right)=\int_{x_{0}}^{x}\left[\mu_{-}\left(w_{+}\left(x^{\prime}\right)-w_{-}\left(x^{\prime}-\beta\right)\right)\right. \\
\left.+\lambda_{-}\left(F_{-}\left(w_{+}\left(x^{\prime}\right), w_{-}\left(x^{\prime}-\beta\right)\right)-w_{-}\left(x^{\prime}-\beta\right)\right)\right] \mathrm{d} x^{\prime} .
\end{array}\right.
$$


Differentiating these two pairs of coupled equations we conclude that the pair $\left\{w_{+}(x), w_{-}(x-\beta)\right\}$ forms a travelling-wave solution with velocity $a_{+}$, and the pair $\left\{w_{+}(x+\beta), w_{-}(x)\right\}$ is a travelling wave with velocity $a_{-}$.

From results of Section 5 (Prop. 5.1) it follows

$$
a_{ \pm} \geq \alpha_{*} c
$$

From (60) we have

$$
a_{ \pm} \leq \alpha_{*} c
$$

Therefore $a_{+}=a_{-}=\alpha_{*} c$ and the theorem is proved.

Remark 6.3. In general, the question whether the limit (62) really exists is still open. Nevertheless it is easy to check (62) at least for isotropic reaction walk.

Proposition 6.4. Let $\mu_{+}=\mu_{-}=\mu, \lambda_{+}=\lambda_{-}=\lambda$ and $J_{11}=J_{22}=J_{12}=J_{21}$. Then

$$
\lim _{\tau \downarrow-\infty} \psi(\tau)=\frac{2 c}{2 \mu+\lambda}
$$

Proof. Let $S$ be the first breeding time. Notice that particles forget its original direction at the breeding time. Hence variables $m_{+}(\tau-S)+X_{+}(S)$ and $m_{-}(\tau-S)+X_{-}(S)$ are identically distributed (for sufficiently large $-\tau)$. Here $X_{+}$and $X_{-}$are the telegraph processes initially moving forwards and backwards respectively. It is easy to see that $\mathbb{E} X_{+}(S)=-\mathbb{E} X_{-}(S)=\frac{c}{\lambda+2 \mu}$. Hence

$$
\lim _{\tau \downarrow-\infty} \psi(\tau)=\frac{2 c}{2 \mu+\lambda}
$$

\section{Appendix A. SOlutions OF LINEAR HYPERBOLIC SYSTEMS}

The objective of this part is to propose the exact formulas for solutions to linear hyperbolic systems and to obtain some inequalities desired in Section 6. The following proposition is well-known (cf. [13]).

Proposition A.1. Solution $v_{ \pm}$of the system

$$
\left\{\begin{array}{l}
-\frac{\partial v_{+}}{\partial \tau}-c \frac{\partial v_{+}}{\partial x}=-b_{11} v_{+}+b_{12} v_{-} \\
-\frac{\partial v_{-}}{\partial \tau}+c \frac{\partial v_{-}}{\partial x}=b_{21} v_{+}-b_{22} v_{-} \\
\tau<t, \quad x \in(-\infty,+\infty)
\end{array}\right.
$$

with terminal conditions $\left.v_{ \pm}\right|_{\tau \uparrow t}=g_{ \pm}(x), x \in(-\infty, \infty)$ has the form

$$
\begin{gathered}
v_{+}=\mathrm{e}^{-b_{11}(t-\tau)} g_{+}(x+c(t-\tau))+\frac{1}{2} e^{-B(t-\tau) / 2} \int_{-(t-\tau)}^{t-\tau} \mathrm{e}^{-b s / 2}\left[b_{12} g_{-}(x+c s) I_{0}\left(\mu_{*} \sqrt{(t-\tau)^{2}-s^{2}}\right)\right. \\
\left.+\mu_{*} g_{+}(x+c s) I_{1}\left(\mu_{*} \sqrt{(t-\tau)^{2}-s^{2}}\right) \frac{t-\tau+s}{\sqrt{(t-\tau)^{2}-s^{2}}}\right] \mathrm{d} s
\end{gathered}
$$


and

$$
\begin{gathered}
v_{-}=\mathrm{e}^{-b_{22}(t-\tau)} g_{-}(x-c(t-\tau))+\frac{1}{2} \mathrm{e}^{-B(t-\tau) / 2} \int_{-(t-\tau)}^{t-\tau} \mathrm{e}^{-b s / 2}\left[b_{21} g_{+}(x+c s) I_{0}\left(\mu_{*} \sqrt{(t-\tau)^{2}-s^{2}}\right)\right. \\
\left.+\mu_{*} g_{-}(x+c s) I_{1}\left(\mu_{*} \sqrt{(t-\tau)^{2}-s^{2}}\right) \frac{t-\tau-s}{\sqrt{(t-\tau)^{2}-s^{2}}}\right] \mathrm{d} s
\end{gathered}
$$

Here $B=b_{11}+b_{22}, b=b_{11}-b_{22}$ and $\mu_{*}=\sqrt{b_{12} b_{21}} ; I_{0}(z)=\sum_{n=0}^{\infty} \frac{z^{2 n}}{2^{2 n}(n !)^{2}}$ is the zero-order Bessel function of imaginary argument, $I_{1}(z)=I_{0}^{\prime}(z)$.

Corollary. For the Heaviside terminal conditions $g_{ \pm}=\theta(-x)$ formulas (A.2)-(A.3) take the form

$$
\begin{gathered}
v_{+}=\mathrm{e}^{-b_{11}(t-\tau)} \theta(-x-c(t-\tau))+\frac{1}{2} \mathrm{e}^{-B(t-\tau) / 2} \int_{-(t-\tau)}^{\min (-x / c, t-\tau)} \mathrm{e}^{-b s / 2}\left[b_{12} I_{0}\left(\mu_{*} \sqrt{(t-\tau)^{2}-s^{2}}\right)\right. \\
\left.+\mu_{*} I_{1}\left(\mu_{*} \sqrt{(t-\tau)^{2}-s^{2}}\right) \frac{t-\tau+s}{\sqrt{(t-\tau)^{2}-s^{2}}}\right] \mathrm{d} s \theta(c(t-\tau)-x) \\
v_{-=} \mathrm{e}^{-b_{22}(t-\tau)} \theta(-x+c(t-\tau))+\frac{1}{2} \mathrm{e}^{-B(t-\tau) / 2} \int_{-(t-\tau)}^{\min (-x / c, t-\tau)} \mathrm{e}^{-b s / 2}\left[b_{21} I_{0}\left(\mu_{*} \sqrt{(t-\tau)^{2}-s^{2}}\right)\right. \\
\left.+\mu_{*} I_{1}\left(\mu_{*} \sqrt{(t-\tau)^{2}-s^{2}}\right) \frac{t-\tau-s}{\sqrt{(t-\tau)^{2}-s^{2}}}\right] \mathrm{d} s \theta(c(t-\tau)-x) .
\end{gathered}
$$

Proposition A2. Let $\left\{v_{+}, v_{-}\right\}$be a solution of (A.1) with Heaviside terminal data $\left.v_{ \pm}\right|_{\tau \uparrow t}=\theta(-x)$. If condition C2 holds, then as $\tau \downarrow-\infty$

$$
v_{ \pm}\left(\alpha_{*} c(t-\tau)-\gamma c \ln (t-\tau), \tau, t\right) \rightarrow 0
$$

where $\alpha_{*}$ is defined by (48).

Proof. Keeping in mind formulas (A.4) and (A.5) it is sufficient to prove that for $x(T)=\alpha_{*} T-\gamma \ln T$

$$
\begin{gathered}
V_{1}(T)=\int_{-T}^{-x(T)} \mathrm{e}^{-b s / 2} I_{0}\left(\mu_{*} \sqrt{T^{2}-s^{2}}\right) \mathrm{d} s=o\left(\mathrm{e}^{B T / 2}\right), \\
V_{2}(T)=\int_{-T}^{-x(T)} \mathrm{e}^{-b s / 2} \frac{\partial}{\partial T} I_{0}\left(\mu_{*} \sqrt{T^{2}-s^{2}}\right) \mathrm{d} s=o\left(\mathrm{e}^{B T / 2}\right)
\end{gathered}
$$

as $T \rightarrow \infty$.

We prove here (A.7) (for (A.8) a similar idea can be applied). We split integral $V_{1}(T)$ into two parts: $V_{1}(T)=V_{11}(T)+V_{12}(T)$, where

$$
V_{11}(T)=\int_{-T}^{-\alpha T} \mathrm{e}^{-b s / 2} I_{0}\left(\mu_{*} \sqrt{T^{2}-s^{2}}\right) \mathrm{d} s, \quad V_{12}(T)=\int_{-\alpha T}^{-x(T)} \mathrm{e}^{-b s / 2} I_{0}\left(\mu_{*} \sqrt{T^{2}-s^{2}}\right) \mathrm{d} s .
$$


Here $\alpha \in\left(\alpha_{*}, 1\right)$. First note that

$$
I_{0}(z) \leq \mathrm{e}^{z}, \quad z \in(-\infty, \infty)
$$

Thus

$$
\begin{gathered}
V_{11} \leq \int_{-T}^{-\alpha T} \exp \left(-\frac{b s}{2}+\mu_{*} \sqrt{T^{2}-s^{2}}\right) \mathrm{d} s \\
=\int_{-T}^{-\alpha T} \exp \left(\mu_{*} T-\frac{b s}{2}-\frac{\mu_{*} s^{2}}{T+\sqrt{T^{2}-s^{2}}}\right) \mathrm{d} s \\
\leq \int_{-T}^{-\alpha T} \exp \left(\mu_{*} T-\frac{b s}{2}-\frac{\mu_{*} s^{2}}{T\left(1+\sqrt{1-\alpha^{2}}\right)}\right) \mathrm{d} s \\
=\exp \left(\mu_{*} T+\frac{b^{2} T}{16 \mu_{*}}\left(1+\sqrt{1-\alpha^{2}}\right)\right) \int_{\beta_{-}}^{\beta_{+}} \exp \left(-\frac{\mu_{*} s^{2}}{T\left(1+\sqrt{1-\alpha^{2}}\right)}\right) \mathrm{d} s
\end{gathered}
$$

where

$$
\beta_{+}=-\alpha T+\frac{b T}{4 \mu_{*}}\left(1+\sqrt{1-\alpha^{2}}\right), \quad \beta_{-}=-T+\frac{b T}{4 \mu_{*}}\left(1+\sqrt{1-\alpha^{2}}\right) .
$$

Note that, if $\alpha_{*}<\alpha<1$, then $\beta_{+}<0$. Indeed, if $b \leq 0$, then it is evident. In the case $b>0$ the inequality $\alpha_{*}>b / B$ is hold (see the proof of Prop. 5.1), and thus by condition C2 $\left(2 \mu_{*}>B\right.$ ) we have

$$
4 \mu_{*} \alpha>4 \mu_{*} \alpha_{*}>4 \mu_{*} \frac{b}{B}>2 b>b\left(1+\sqrt{1-\alpha^{2}}\right)
$$

which is desired.

By the inequality

$$
\int_{-\infty}^{-A} \mathrm{e}^{-x^{2} / 2 \sigma^{2}} d x \leq \frac{\sigma^{2}}{A^{2}} \mathrm{e}^{-A^{2} / 2 \sigma^{2}}, \quad A>0
$$

the right hand side of (A.9) can be estimated by

$$
\text { const } \cdot \exp \left(\frac{b \alpha T}{2}+\mu_{*} T \sqrt{1-\alpha^{2}}\right)=o\left(\mathrm{e}^{B T / 2}\right)
$$

(see $(47))$.

To estimate $V_{12}$ we apply the inequality

$$
I_{0}(z) \leq \frac{\mathrm{e}^{z}}{\sqrt{2 \pi z}}, \quad z \rightarrow \infty
$$

Thus

$$
V_{12} \leq \frac{1}{\left(2 \pi \mu_{*} T\right)^{1 / 2}\left(1-\alpha^{2}\right)^{1 / 4}} \int_{-\alpha T}^{-\alpha_{*} T+\gamma \ln T} \exp \left(-\frac{b s}{2}+\mu_{*} \sqrt{T^{2}-s^{2}}\right) \mathrm{d} s .
$$

In the same way as before one can obtain

$$
V_{12} \leq \mathrm{const} \cdot T^{-1 / 2} \exp \left(\frac{b \alpha_{*} T}{2}-\frac{b \gamma \ln T}{2}+\mu_{*} \sqrt{T^{2}-\left(-\alpha_{*} T+\gamma \ln T\right)^{2}}\right)
$$




$$
\begin{gathered}
\leq \text { const } \cdot T^{-1 / 2} \exp \left(\frac{b \alpha_{*} T}{2}+\mu_{*} T \sqrt{1-\alpha_{*}^{2}}-\frac{b \gamma \ln T}{2}+\frac{\mu_{*} \alpha_{*} \gamma \ln T}{\sqrt{1-\alpha_{*}^{2}}}\right) \\
=\text { const } \cdot T^{-1 / 2+\gamma\left(-b / 2+\mu_{*} \alpha_{*} / \sqrt{1-\alpha_{*}^{2}}\right)} \exp \left(\frac{b \alpha_{*} T}{2}+\mu_{*} T \sqrt{1-\alpha_{*}^{2}}\right) \\
=\delta_{T} e^{B T / 2}
\end{gathered}
$$

where $\delta_{T}=$ const $\cdot T^{-1 / 2+\gamma\left(-b / 2+\mu_{*} \alpha_{*} / \sqrt{1-\alpha_{*}^{2}}\right)} \rightarrow 0$ for suitably chosen positive $\gamma$. In the last line above we use (52). Consequently, property (A.7) follows from this and from (A.10). The proposition is proved.

\section{Appendix B. Existence And uniqueness Results}

We shall prove here the existence and uniqueness of the solution of system (5) with measurable terminal data $g_{ \pm}$.

First notice that the linear system

$$
\left\{\begin{array}{l}
-\frac{\partial v_{+}}{\partial \tau}-c \frac{\partial v_{+}}{\partial x}=\mu_{+}\left(v_{-}-v_{+}\right)-\lambda_{+} v_{+}, \\
-\frac{\partial v_{-}}{\partial \tau}+c \frac{\partial v_{-}}{\partial x}=\mu_{-}\left(v_{+}-v_{-}\right)-\lambda_{-} v_{-}
\end{array} \quad \tau<t\right.
$$

(with the terminal condition $\left.v_{ \pm}\right|_{\tau \uparrow t}=g_{ \pm}, g_{ \pm} \in \mathcal{C}^{1}$ ) has the unique solution in $\mathcal{C}^{1}$. Moreover this solution can be represented in the Fenmann-Kac form (see Th. 2.1)

$$
v_{ \pm}(x, \tau, t)=\mathbb{E}_{ \pm,(x, \tau)}\left[g(X(t), \sigma(t), t) \exp \left(-\int_{\tau}^{t} \lambda_{\sigma(s)} \mathrm{d} s\right)\right]:=U_{\tau, t}(g)
$$

where $(X, \sigma)$ is the telegraph process with intensities $\mu_{ \pm}$. Fix $t, t \in(-\infty, \infty)$. Solution $v=U_{\tau, t}(g), \tau \leq t$ forms a semigroup acting on $\mathcal{C}^{1} \times \mathcal{C}^{1}$.

From (B.2) it follows the monotonicity of semigroup $U$ : if $f_{+} \leq g_{+}$and $f_{-} \leq g_{-}$, then $U_{\tau, t}(f)(x, \sigma) \leq$ $U_{\tau, t}(g)(x, \sigma),-\infty<x<\infty, \sigma= \pm$. Moreover, if $g(x, \sigma) \leq C$, then $U_{\tau, t}(g)(x, \sigma) \leq C \mathbb{E}_{\sigma} \exp \left(-\int_{\tau}^{t} \lambda_{\sigma(s)} \mathrm{d} s\right)$

By the Riezs representation theorem semigroup $U_{\tau, t}, \tau \leq t$ has a canonical extension on $\mathcal{B} \times \mathcal{B}$, the space of bounded measurable functions. In particular, if function $g$ is peace-wise continues, then the solution $v=$ $v(x, \sigma, \tau, t)=U_{\tau, t}(g)(x, \sigma)$ has discontinuities propagating along characteristics.

We regard solution $u$ of non-linear system (5) as a weak solution. It means that for $\tau<t$ and a test function $\varphi=\varphi(x, \sigma, \tau), \varphi_{ \pm} \in \mathcal{C}_{0}^{1}$

$$
\left\{\begin{array}{l}
\int_{\tau}^{t} \int u_{+}(x, s, t)\left[\frac{\partial \varphi_{+}}{\partial s}+c \frac{\partial \varphi_{+}}{\partial x}+\left(\mu_{+}+\lambda_{+}\right) \varphi_{+}\right](x, s) \mathrm{d} s \mathrm{~d} x-\mu_{+} \int_{\tau}^{t} \int u_{-}(x, s, t) \varphi_{+}(x, s) \mathrm{d} s \mathrm{~d} x \\
-\lambda_{+} \int_{\tau}^{t} \int F_{+}\left(u_{+}, u_{-}\right) \varphi_{+}(x, s) \mathrm{d} s \mathrm{~d} x=\int u_{+}(x, \tau) \varphi_{+}(x, \tau) \mathrm{d} x-\int g_{+}(x, t) \varphi_{+}(x, t) \mathrm{d} x, \\
\int_{\tau}^{t} \int u_{-}(x, s, t)\left[\frac{\partial \varphi_{-}}{\partial s}-c \frac{\partial \varphi_{-}}{\partial x}+\left(\mu_{-}+\lambda_{-}\right) \varphi_{-}\right](x, s) \mathrm{d} s \mathrm{~d} x-\mu_{-} \int_{\tau}^{t} \int u_{+}(x, s, t) \varphi_{-}(x, s) \mathrm{d} s \mathrm{~d} x \\
-\lambda_{-} \int_{\tau}^{t} \int F_{-}\left(u_{+}, u_{-}\right) \varphi_{-}(x, s) \mathrm{d} s \mathrm{~d} x=\int u_{-}(x, \tau) \varphi_{-}(x, \tau) \mathrm{d} x-\int g_{-}(x, t) \varphi_{-}(x, t) \mathrm{d} x .
\end{array}\right.
$$


Theorem B.1. If $u$ is the solution of system (B.3), then $u$ satisfies

$$
u=U_{\tau, t}(g)-\int_{\tau}^{t} U_{t+\tau-s, t}\left(\left.\lambda F(u)\right|_{s}\right) \mathrm{d} s
$$

for for each $\tau<t$ and for almost every $x$.

Proof. Consider the system

$$
\left\{\begin{array}{l}
-\frac{\partial \psi_{+}}{\partial s}+c \frac{\partial \psi_{+}}{\partial x}=-\left(\mu_{+}+\lambda_{+}\right) \psi_{+}+\mu_{-} \psi_{-}, \\
-\frac{\partial \psi_{-}}{\partial s}-c \frac{\partial \psi_{-}}{\partial x}=-\left(\mu_{-}+\lambda_{-}\right) \psi_{-}+\mu_{+} \psi_{+}
\end{array} \quad s<t\right.
$$

with the terminal condition $\left.\psi_{ \pm}\right|_{s \uparrow t}=\psi_{ \pm}^{0}, \psi_{ \pm}^{0} \in \mathcal{C}_{0}^{\infty}$.

Solution $\psi_{ \pm}, \psi(x, s, t)=U_{s, t}^{*}\left(\psi^{0}\right)$ of this system is defined by semigroup $U_{s, t}^{*}$ dual to $U_{s, t}$. By the statement that $U_{\tau, t}$ and $U_{\tau, t}^{*}$ are dual we mean that for $\tau<t$ and bounded measurable functions $g, \psi$

$$
\int g(x, t) \cdot U_{\tau, t}^{*}(\psi)(x) \mathrm{d} x=\int U_{\tau, t}(g)(x) \cdot \psi(x, t) \mathrm{d} x
$$

where $f \cdot g=f_{+} g_{+}+f_{-} g_{-}$. To finish the of Theorem it is sufficient to substitute $\varphi(x, s)=\psi(x, t+\tau-s)$ in system (B.3).

Notice that the solution of system (B.5) can be expressed by means of the reversed underlying telegraph process $X^{*}(s)=X(t-s)$. More precisely,

$$
\varphi_{ \pm}(x, s)=\mathbb{E}\left[\psi^{0}\left(X^{*}(s), \sigma^{*}(t), t\right) \exp \left(-\int_{s}^{t} \lambda_{\sigma^{*}\left(s^{\prime}\right)} \mathrm{d} s^{\prime}\right) \mid \sigma^{*}(s)= \pm, X^{*}(s)=x\right] .
$$

Theorem B.2. Let $0 \leq g_{ \pm} \leq 1, g \in \mathcal{B} \times \mathcal{B}$. Then the solution $u \in \mathcal{B} \times \mathcal{B}, 0 \leq u_{ \pm} \leq 1$ of equation (B.4) exists and it is unique.

Proof. Define

$$
\begin{gathered}
u^{(0)}=U_{\tau, t}(g) \\
u^{(n)}=U_{\tau, t}(g)-\int_{\tau}^{t} U_{t+\tau-s, t}\left(\left.\lambda F\left(u^{(n-1)}\right)\right|_{s}\right) \mathrm{d} s, \quad n \geq 1 .
\end{gathered}
$$

Then, applying the usual contraction technique, one can prove the convergence $u^{(n)} \rightarrow u$ uniformly on $(\tau, t]$, which gives the exact solution of equation (B.4).

Acknowledgements. The author is grateful to the remarks of two anonymous referees.

\section{REFERENCES}

[1] K.B. Athreya and P.E. Ney, Branching processes. Dover Publ. Inc. Mineola, NY (2004).

[2] L. Beghin, L. Nieddu and E. Orsingher, Probabilistic analysis of the telegrapher's process with drift by mean of relativistic transformations. J. Appl. Math. Stoch. Anal. 14 (2001) 11-25.

[3] M. Bramson, Maximal displacement of branching Brownian motion. Comm. Pure Appl. Math. 31 (1978) 531-581.

[4] M. Bramson, Convergence of solutions of the Kolmogorov equation to travelling waves. Mem. Amer. Math. Soc. 44 (1983) iv +190 . 
[5] B. Chauvin and A. Rouault, Supercritical branching Brownian motion and K-P-P equation in the critical speed-are. Math. Nachr. 19 (1990) 41-59.

[6] A. Di Crescenzo and F. Pellerey, On prices' evolutions based on geometric telegrapher's process. Appl. Stoch. Models Business Industry 18 (2002) 171-184.

[7] S.R. Dunbar, A branching random evolution and a nonlinear hyperbolic equation. SIAM J. Appl. Math. 48 (1988) 1510-1526.

[8] S.R. Dunbar and H.G. Othmer, On a nonlinear hyperbolic equation describing transmission lines, cell movement, and branching random walks, in Nonlinear oscillations in biology and chemistry, (Salt Lake City, Utah, 1985). Lect. Notes Biomath. 66 (1986) $274-289$.

[9] R.A. Fisher, The advance of advantageous genes. Ann. Eugenics 7 (1937) 335-369.

[10] J. Fort and V. Mendez, Wavefronts in time-delayed reaction-diffusion systems. Theory and comparison to experiment. Rep. Prog. Phys. 65 (2002) 895-954.

[11] S. Goldstein, On diffusion by discontinuous movements and on the telegraph equation. Quart. J. Mech. Apl. Math. 4 (1951) $129-156$.

[12] K.P. Hadeler, Nonlinear propagation in reaction transport systems. Differential equations with applications to biology, Halifax, NS, 1997, Fields Inst. Commun. 21 Amer. Math. Soc., Providence, RI (1999) 251-257.

[13] K.P. Hadeler, Reaction transport systems in biological modelling, In Mathematics inspiring by biology. Lect. Notes in Math. 1714 (1999) 95-150.

[14] K.P. Hadeler, T. Hillen and F. Lutscher, The Langevin or Kramer approach to biological modelling. Math. Mod. Meth. Appl. Sci. 14 (2004) 1561-1583.

[15] T. Hillen and H.G. Othmer, The diffusion limit of transport equations derived from velocity-jump processes. SIAM J. Appl. Math. 61 (2000) 751-775.

H.G. Othmer and T. Hillen, The diffusion limit of transport equations. II. Chemotaxis equations. SIAM J. Appl. Math. 62, (2002) 1222-1250

[16] W. Horsthemke, Spatial instabilities in reaction random walks with direction-independent kinetics. Phys. Rev. E 60 (1999) 2651-2663.

[17] W. Horsthemke, Fisher waves in reaction random walks. Phys. Lett. A 263 (1999) $285-292$.

[18] D.D. Joseph and L. Preziosi, Heat waves. Rev. Mod. Phys. 61 (1989) 41-73.

[19] D.D. Joseph and L. Preziosi, Addendum to the paper "Heat waves". Rev. Mod. Phys. 62 (1990) $375-391$.

[20] M. Kac, Probability and related topics in physical sciences. Interscience, London (1959).

[21] M. Kac, A Stochastic model related to the telegraph equation. Rocky Mountain J. Math. 4 (1974) $497-509$.

[22] A. Kolmogorov, I. Petrovskii and N. Piskunov, Étude de l'équation de la diffusion avec croissance de la quantité de la matière et son application à un problème biologique. Bull. Math. 1 (1937) 1-25.

[23] O.D. Lyne, Travelling waves for a certain first-order coupled PDE system. Electronic J. Prob. 5 (2000) 1-40.

[24] O.D. Lyne and D. Williams, Weak solutions for a simple hyperbolic system. Electronic J. Prob. 6 (2001) 1-21.

[25] H.P. McKean, Application of Brownian motion to the equation of Kolmogorov-Petrovskii-Piskunov. Comm. Pure Appl. Math. XXVIII (1975) 323-331

[26] H.P. McKean, Correction to above. Comm. Pure Appl. Math. XXIX (1976) 553-554.

[27] S. Mizohata, The theory of partial differential equations. Cambridge University Press, New York (1973) xii+490.

[28] V. Mendez and J. Camacho, Dynamics and thermodynamics of delayed population growth. Phys. Rev. E 55 (1997) 6476-6482.

[29] V. Mendez and A. Compte, Wavefronts in bistable hyperbolic reaction-diffusion systems. Physica A 260 (1998) 90-98.

[30] M. Nagasawa, Schrödinger equations and diffusion theory. Monographs in Mathematics, Birkhäuser Verlag, Basel 86 (1993) pp. $\mathrm{x}+319$

[31] E. Orsingher, Probability law, flow function, maximum distribution of wave governed random motions and their connections with Kirchoff's laws. Stoch. Proc. Appl. 34 (1990) 49-66.

[32] H.G. Othmer, S.R. Dunbar and W. Alt, Models of dispersal in biological systems. J. Math. Biol. 26 (1988) 263-298.

[33] M. Pinsky, Lectures on random evolution. World Scient. Publ. Co., River Edge, NY (1991).

[34] N.E. Ratanov, Telegraph processes with reflecting and absorbing barriers in inhomogeneous media. Theor. Math. Phys. 112 (1997) 857-865

[35] N. Ratanov, Reaction-advection random motions in inhomogeneous media. Physica D 189 (2004) 130-140.

[36] N. Ratanov, Pricing options under telegraph processes. Rev. Econ. Ros. 8 (2005) 131-150.

[37] A.I. Volpert, V.A. Volpert and Vl.A. Volpert, Travelling wave solutions of parabolic systems. Translated from the Russian manuscript by James F. Heyda. Translations of Mathematical Monographs. 140 Amer. Math. Soc. Providence, RI, (1994) pp. $\mathrm{xii}+448$.

[38] G.H. Weiss, Aspects and applications of the random walk. North-Holland, Amsterdam (1994).

[39] G.H. Weiss, Some applications of persistent random walks and the telegrapher's equation. Physica A 311 (2002) 381-410. 